\title{
Combined longitudinal and transverse noise enhancement in lasers
}

\author{
Krista Joosten and Gerard Nienhuis \\ Huygens Laboratorium, Rijksuniversiteit Leiden, Postbus 9504, 2300 RA Leiden, The Netherlands
}

(Received 24 June 1998)

\begin{abstract}
Using a semiclassical approach we derive a general expression for the quantum-limited linewidth of a single-mode laser. We include both nonuniform properties of the laser medium and localized losses at mirrors and apertures. For such systems the transverse modes are known to be nonorthogonal, giving rise to an enhancement of the laser noise. The transverse factor varies, in general, along the propagation direction. The combination of transverse and longitudinal noise enhancement is far from trivial. In particular, we show that for an aperture in the cavity, the transverse excess noise factor is the geometric mean of the factors pertaining to the two regions in which the aperture divides the cavity. [S1050-2947(98)06112-5]

PACS number(s): 42.60.Da, 42.50.Lc
\end{abstract}

\section{INTRODUCTION}

Spontaneous emission is the fundamental noise source in lasers. In the ideal case of small output coupling, negligible internal losses, and uniform field intensity, the SchawlowTownes (ST) limit holds, and the laser linewidth is given by

$$
\Delta \omega_{\mathrm{ST}}=\frac{\hbar \omega \Gamma_{0}^{2}}{2 \mathcal{P}_{\mathrm{out}}} .
$$

Here, $\mathcal{P}_{\text {out }}$ is the laser output power and $\Gamma_{0}$ is the decay rate of the laser cavity [1]. In the presence of nonuniform loss or gain, the propagation operator describing the field traversing the laser cavity is nonunitary. This generally destroys the orthogonality of the laser modes. This nonorthogonality basically arises from loss-induced mode coupling. It can be demonstrated [2] that this leads to an enhancement of spontaneous-emission noise in the lasing mode. A first discussion of the effect was given by Petermann [3] in the special case of gain-guided semiconductor lasers. The nonuniform gain profile is equivalent to an imaginary potential in the Schrödinger equation. It causes the transverse modes, $u_{n}(\rho)$, to obey a modified orthogonality condition $\int d \rho u_{n} u_{m}=\delta_{n m}$ in terms of a scalar product without a complex conjugate. [Throughout this paper, we denote as $\rho$ $=(x, y)$ the transverse coordinates of a light field propagating in the $z$ direction.] The excess noise factor $K$ arises when expanding the spontaneous-emission field in the set of modes which are nonorthogonal in the usual sense. When the lasing mode is $u_{n}(\rho)$, this factor is [3]

$$
K_{T}=\frac{\left|\int d \rho u_{n}^{\star}(\rho) u_{n}(\rho)\right|^{2}}{\left|\int d \rho u_{n}^{2}(\rho)\right|^{2}} .
$$

The property of nonorthogonality of the transverse modes is not restricted to gain-guided semiconductor lasers. In general, the set $\left\{u_{n}(\rho)\right\}$ will be nonorthogonal in the presence of nonuniform gain or losses in the transverse direction, thus leading to an enhancement in the noise [2]. Large transverse nonorthogonalities, for example, can arise from spillover at the end mirrors of a laser, such as occurs in unstable cavities
[4-6]. It has been shown that even for stable laser resonators large excess noise is possible when apertures give rise to large diffraction losses [7-9].

In a similar fashion, a noise-enhancement factor arises when the noise field from spontaneous emission acquires a varying amplitude during propagation through the laser cavity during a round trip, due to the combined action of gain and loss [10-12]. In the absence of gain saturation, the corresponding enhancement factor can likewise be expressed in terms of the overlap of nonorthogonal longitudinal modes [13]. For a uniform medium, the longitudinal factor is [14]

$$
K_{L}=\left[\frac{\left(\sqrt{R_{1}}+\sqrt{R_{2}}\right)\left(1-\sqrt{R_{1} R_{2}}\right)}{\sqrt{R_{1} R_{2}} \ln \left(R_{1} R_{2}\right)}\right]^{2}
$$

with $R_{1}$ and $R_{2}$ the intensity reflectivities of the two end mirrors. Theories describing the effect of axially inhomogeneous media on the laser linewidth have been developed by several groups $[10,15,16]$. The excess noise factor has been studied experimentally, both for longitudinal $[17,15]$ and transverse nonorthogonality [18-20].

The combination of transverse and longitudinal contributions to the enhancement of spontaneous-emission noise has also received some attention. When the field distribution is the product of a transverse and a longitudinal distribution, the noise enhancement is well described by the product $K_{T} K_{L}$ [10]. When the laser waveguide and the gain are uniform in the longitudinal direction, a generalized expression for the enhancement factor in terms of three-dimensional overlap integrals of the field distribution has been justified $[21,22]$. For the case that the gain and loss coefficients vary in this direction, an expression for the enhancement factor has been derived in terms of integrals involving the positiondependent material coefficients [23].

In the present paper we discuss the effect of longitudinal and transverse inhomogeneities on the excess noise factor. In particular, the effect of optical elements such as apertures is considered. We allow for apertures inside the resonator, which may divide the laser medium in two parts with a different transverse excess noise factor. We take advantage of the close analogy between the propagation of light beams and the evolution of a wave packet in quantum mechanics. 

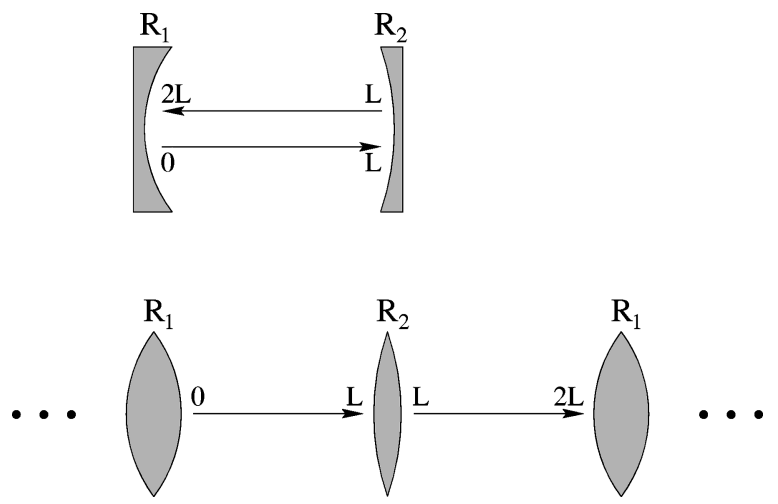

FIG. 1. Cavity and equivalent lens guide.

\section{PARAXIAL WAVE EQUATION}

We describe the laser cavity as a resonator filled with a medium with a dielectric constant $\varepsilon(\omega, \vec{r})$ that depends on both frequency and position. It is convenient to describe the oscillating field in a standing-wave resonator as a traveling wave in the corresponding unfolded periodic lens guide, when each of the end mirrors with finite reflectivity $R$ is replaced by a lens with transmittivity $R[24,2,25]$. In this way, a standing-wave cavity with length $L$ is replaced by a lens guide with period $2 L$ as sketched in Fig. 1 . Here we have assumed that the cavity contains no reflecting optical elements in between the end mirrors. The case of a traveling wave in a ring laser can be described by the same model system, where $2 L$ is the round-trip distance.

Starting from Maxwell's equation

$$
\vec{\nabla} \times(\vec{\nabla} \times \vec{E})=-\mu_{0} \partial_{t}^{2} \vec{D}
$$

we make the paraxial approximation by substituting for a light beam propagating in the positive $z$ direction

$$
\vec{E}(\vec{r}, t)=\operatorname{Re} \vec{e} E_{\rightarrow}(\vec{r}, t) e^{i(k z-\omega t)} .
$$

Polarization effects are ignored by assuming $\vec{e}$ to be uniform. The amplitude $E_{\rightarrow}$ is supposed to vary slowly as a function of $z$ and $t$, so that its second derivative can be neglected. Hence we may write

$$
\vec{\nabla} \times(\vec{\nabla} \times \vec{E})=\operatorname{Re} \vec{e} e^{i(k z-\omega t)}\left(k^{2} E_{\rightarrow}-2 i k \partial_{z} E_{\rightarrow}-\partial_{\rho}^{2} E_{\rightarrow}\right) .
$$

When the dielectric constant $\varepsilon$ varies little over the bandwidth of the light field, the $n$th time derivative of the displacement can be expressed as

$$
\begin{aligned}
\partial_{t}^{n} \vec{D}= & \varepsilon_{0} \operatorname{Re} \vec{e}\left[(-i \omega)^{n} \varepsilon(\omega) E_{\rightarrow}(t)\right. \\
& \left.+(-i)^{n-1} \partial_{\omega}\left(\omega^{n} \varepsilon(\omega)\right) \partial_{t} E_{\rightarrow}(t)\right] e^{i(k z-\omega t)}
\end{aligned}
$$

This generalizes the result derived by Milonni in the case $n$ $=1$ [26]. Substituting Eqs. (2.3) and (2.4) for $n=2$ into Eq. (2.1) gives the paraxial wave equation for a light beam traveling to the right down the effective lens guide. We find

$$
\partial_{z} E_{\rightarrow}=-i \hat{H} E_{\rightarrow}-\frac{\omega}{c^{2} k} \varepsilon_{\mathrm{gr}}(\omega) \partial_{t} E_{\rightarrow} .
$$

Here $\hat{H}$ is an effective Hamilton operator, defined by

$$
\hat{H} E_{\rightarrow}=-\frac{1}{2 k} \partial_{\rho}^{2} E_{\rightarrow}+\frac{k}{2}\left(1-\frac{\omega^{2} \varepsilon}{c^{2} k^{2}}\right) E_{\rightarrow} .
$$

In Eq. (2.5) we used the notation $\varepsilon_{\mathrm{gr}}=\varepsilon+\frac{1}{2} \omega \partial_{\omega} \varepsilon$ for the group refractive index at the field frequency. Notice that the group velocity is given by $v_{\mathrm{gr}}=c \sqrt{\varepsilon} / \varepsilon_{\mathrm{gr}}$. For simplicity we will assume $\varepsilon_{\text {gr }}$ and the group velocity to be real. The (real) value of $\omega$ and $k$ can be selected such that $c k / \omega$ is equal to some average of the real refractive index of the medium. In the steady state, where $\partial_{t} E_{\rightarrow}=0$, the wave equation (2.5) is identical in form to the time-dependent Schrödinger equation. The propagation coordinate $z$ plays the role of time, and the transverse coordinate mimics position. A large dielectric constant serves as a potential well. Since we allow $\varepsilon(\omega)$ to be complex, the effective potential is also complex in general. A positive imaginary part represents losses, and gain corresponds to a negative imaginary part of $\varepsilon(\omega)$. Index or gain guiding can be expressed by the $\rho$ dependence of $\varepsilon$. The time derivative in Eq. (2.5) ensures that a localized wave packet propagates at the group velocity.

In the same way, a beam propagating in the negative $z$ direction can be described by substituting

$$
\vec{E}(\vec{r}, t)=\operatorname{Re} \vec{e} E_{\leftarrow}(\vec{r}, t) e^{-i(k z+\omega t)}
$$

into Maxwell's equation. This leads to the paraxial wave equation

$$
\partial_{z} E_{\leftarrow}=i \hat{H} E_{\leftarrow}+\frac{\omega}{c^{2} k} \varepsilon_{\mathrm{gr}}(\omega) \partial_{t} E_{\leftarrow} .
$$

The effect of optical elements, such as mirrors, lenses, and apertures, can be described by a multiplicative factor $\chi(\rho)$. When $z_{+}$is a position just to the right and $z_{-}$is to the left of the element's position, we can write

$$
\begin{aligned}
& E_{\rightarrow}\left(z_{+}, \rho\right)=\chi(\rho) E_{\rightarrow}\left(z_{-}, \rho\right), \\
& E_{\leftarrow}\left(z_{-}, \rho\right)=\chi(\rho) E_{\leftarrow}\left(z_{+}, \rho\right) .
\end{aligned}
$$

For a nonabsorbing lens, or a perfectly reflecting mirror, the factor $\chi$ has absolute value 1 , so that it only applies a phase factor to the beam. A hard-edged aperture is modeled simply by setting $\chi=1$ for a transverse coordinate $\rho$ within the opening and $\chi=0$ outside it.

For convenience we represent the transverse field distribution $E_{\rightarrow}(\rho, z)$ or $E_{\leftarrow}(\rho, z)$ as a state vector, which we denote as $\left|E_{\rightarrow}(z)\right\rangle$ or $\left|E_{\leftarrow}(z)\right\rangle$, just as in quantum mechanics. The fields may be viewed as the wave functions in coordinate representation, so that $E_{\rightarrow}(z, \rho)=\left\langle\rho \mid E_{\rightarrow}(z)\right\rangle$ and $E_{\leftarrow}(z, \rho)=\left\langle\rho \mid E_{\leftarrow}(z)\right\rangle$. Then the propagation of a light beam can be expressed in terms of propagation operators $[27,2]$. In the steady state, the wave equations (2.5) and (2.8) read 


$$
\begin{gathered}
\frac{d}{d z}\left|E_{\rightarrow}(z)\right\rangle=-i \hat{H}(z)\left|E_{\rightarrow}(z)\right\rangle, \\
\frac{d}{d z}\left|E_{\leftarrow}(z)\right\rangle=i \hat{H}(z)\left|E_{\leftarrow}(z)\right\rangle,
\end{gathered}
$$

where the $z$ dependence of the Hamiltonian arises from the variation of $\varepsilon$ in the propagation direction. The transformation of the state vectors across an optical element can likewise be expressed as a linear operator. Obviously, in the presence of absorption this operator is not unitary, and when it blocks the light completely outside the opening, the operator is not invertible: knowledge of $\left|E_{\rightarrow}\left(z_{+}\right)\right\rangle$is not sufficient to reconstruct $\left|E_{\rightarrow}\left(z_{-}\right)\right\rangle$.

When the transverse field pattern $E_{\rightarrow}\left(z_{1}, \rho\right)$, or equivalently the state vector $\left|E_{\rightarrow}\left(z_{1}\right)\right\rangle$, is known for a given value of $z_{1}$, the field pattern for all values $z_{2}>z_{1}$ follows from the evolution equation (2.10), combined with the transformation operators across all optical elements between $z_{1}$ and $z_{2}$. The relation can be expressed in terms of a linear propagation operator $\hat{O}_{\rightarrow}\left(z_{2}, z_{1}\right)$, so that

$$
\left|E_{\rightarrow}\left(z_{2}\right)\right\rangle=\hat{O}_{\rightarrow}\left(z_{2}, z_{1}\right)\left|E_{\rightarrow}\left(z_{1}\right)\right\rangle .
$$

Likewise, propagation to the left can be written as

$$
\left|E_{\leftarrow}\left(z_{1}\right)\right\rangle=\hat{O}_{\leftarrow}\left(z_{1}, z_{2}\right)\left|E_{\leftarrow}\left(z_{2}\right)\right\rangle .
$$

Then the operators $\hat{O}_{\rightarrow}$ and $\hat{O}_{\leftarrow}$ obey the propagation operations

$$
\begin{gathered}
\frac{d}{d z_{2}} \hat{O}_{\rightarrow}\left(z_{2}, z_{1}\right)=-i \hat{H}\left(z_{2}\right) \hat{O}_{\rightarrow}\left(z_{2}, z_{1}\right), \\
\frac{d}{d z_{1}} \hat{O}_{\leftarrow}\left(z_{1}, z_{2}\right)=i \hat{H}\left(z_{1}\right) \hat{O}_{\leftarrow}\left(z_{1}, z_{2}\right)
\end{gathered}
$$

in between optical elements, and their transformation over an optical element is determined by the operator $\hat{\chi}$. In the infinitesimal transformation across an optical element, this gives

$$
\begin{aligned}
\left\langle\rho\left|\hat{O}_{\rightarrow}\left(z_{+}, z_{-}\right)\right| \rho^{\prime}\right\rangle & =\delta_{2}\left(\rho-\rho^{\prime}\right) \chi(\rho) \\
& =\left\langle\rho\left|\hat{O}_{\leftarrow}\left(z_{-}, z_{+}\right)\right| \rho^{\prime}\right\rangle .
\end{aligned}
$$

The boundary conditions are $\hat{O}_{\rightarrow}\left(z_{1}, z_{1}\right)=\hat{I}=\hat{O}_{\leftarrow}\left(z_{2}, z_{2}\right)$, with $\hat{I}$ the unit operator.

In the presence of absorption or gain, the Hamiltonian $\hat{H}$ is not Hermitian, and each $\hat{O}$ is not unitary. On the other hand, since the non-Hermiticity of the Hamiltonian results only from the complex effective potential, it is easy to verify that $\hat{H}=\hat{H}^{T}$, where the transpose operator $\hat{H}^{T}$ is defined by

$$
\left\langle\rho\left|\hat{H}^{T}(z)\right| \rho^{\prime}\right\rangle=\left\langle\rho\left|\hat{H}^{\dagger}(z)\right| \rho^{\prime}\right\rangle^{\star}=\left\langle\rho^{\prime}|\hat{H}(z)| \rho\right\rangle .
$$

For the propagation operators, this gives the relation

$$
\hat{O}_{\leftarrow}\left(z_{1}, z_{2}\right)=\left[\hat{O}_{\rightarrow}\left(z_{2}, z_{1}\right)\right]^{T} .
$$

In coordinate representation, these operators give the propagation kernels for propagation to the left or to the right, with the relation

$$
\begin{aligned}
K_{\rightarrow}\left(z_{2}, \rho_{2} ; z_{1}, \rho_{1}\right) & =\left\langle\rho_{2}\left|\hat{O}_{\rightarrow}\left(z_{2}, z_{1}\right)\right| \rho_{1}\right\rangle \\
& =\left\langle\rho_{1}\left|\hat{O}_{\leftarrow}\left(z_{1}, z_{2}\right)\right| \rho_{2}\right\rangle \\
& =K_{\leftarrow}\left(z_{1}, \rho_{1} ; z_{2}, \rho_{2}\right) .
\end{aligned}
$$

Here we assumed that the optical elements and the dielectric constant $\varepsilon$ are independent of the propagation direction. This relation, for example, would not be satisfied in the presence of Doppler broadening in a lens guide with a flowing gain medium.

Relation (2.16) between the propagation operators $\hat{O}_{\rightarrow}$ and $\hat{O}_{\leftarrow}$ determines the biorthogonality relation between the eigenmodes propagating in the two directions, as will become clear in the subsequent section. To conclude the present section, we point out that the overlap of the transverse field patterns corresponding to the light beams $E_{\rightarrow}$ and $E_{\leftarrow}$ is independent of the longitudinal coordinate $z$. By using the wave equations (2.10), one readily checks that

$$
\frac{d}{d z}\left\langle E_{\leftarrow}^{\star}(z) \mid E_{\rightarrow}(z)\right\rangle=0,
$$

which implies that the integral $\int d \rho E_{\leftarrow}(z, \rho) E_{\rightarrow}(z, \rho)$ is independent of $z$. This relation is valid under quite general conditions for counterpropagating beams through media and optical elements with arbitrary transverse inhomogeneity.

When the lens-guide model is used to represent a standing-wave cavity with length $L$, a light wave at position $z$ traveling to the right is physically identical to a wave at position $2 L-z$ traveling to the left. This implies that

$$
\hat{O}_{\rightarrow}\left(z_{2}, z_{1}\right)=\hat{O}_{\leftarrow}\left(2 L-z_{2}, 2 L-z_{1}\right), \quad z_{2}>z_{1} .
$$

In the case of a ring laser, beams propagating in opposite directions are physically different.

\section{TRANSVERSE EIGENMODES}

Since the lens guide models a periodic structure, two points $z$ and $z+2 L$ that are separated by a period are physically equivalent. It is therefore natural to consider light waves that are self-reproducing after propagation over one period. These are the transverse eigenmodes of the laser cavity [2]. When we arbitrarily select a reference plane at the propagation coordinate $z=z_{0}$, the round-trip propagation operators over one period starting from this reference plane are

$$
\hat{O}_{\rightarrow, 0}=\hat{O}_{\rightarrow}\left(z_{0}+2 L, z_{0}\right), \quad \hat{O}_{\leftarrow, 0}=\hat{O}_{\leftarrow}\left(z_{0}, z_{0}+2 L\right) .
$$

From Eq. (2.16) it follows that these two operators are each other's transpose, so that $\hat{O}_{\leftarrow, 0}=\hat{O}_{\rightarrow, 0}^{T}$. The transverse eigenmodes of the system propagating to the right are the right-hand eigenmodes of $\hat{O}_{\rightarrow, 0}$, defined by the eigenvalue relation

$$
\hat{O}_{\rightarrow, 0}\left|u_{n}\right\rangle=\gamma_{n}\left|u_{n}\right\rangle,
$$


and the corresponding transverse eigenmode propagating to the left obeys the analogous relation

$$
\hat{O}_{\leftarrow, 0}\left|f_{n}\right\rangle=\gamma_{n}\left|f_{n}\right\rangle .
$$

It will become clear in a moment that the set of eigenvalues $\gamma_{n}$ is the same for both operators. Since these operators are not unitary, one cannot expect that the eigenvectors are orthogonal, or that the eigenvalues are unitary. In general, these operators have left-hand eigenvectors with the same eigenvalues, obeying the equalities

$$
\left\langle v_{n}\right| \hat{O}_{\rightarrow, 0}=\left\langle v_{n}\right| \gamma_{n}, \quad\left\langle g_{n}\right| \hat{O}_{\leftarrow, 0}=\left\langle g_{n}\right| \gamma_{n} .
$$

A left eigenvector is orthogonal to a right eigenvector at a different eigenvalue, and normalization can be chosen so that the left and right eigenmodes obey the biorthonormality condition

$$
\left\langle v_{n} \mid u_{n^{\prime}}\right\rangle=\delta_{n n^{\prime}}, \quad\left\langle g_{n} \mid f_{n^{\prime}}\right\rangle=\delta_{n n^{\prime}} .
$$

Moreover, since $\hat{O}_{\leftarrow, 0}=\hat{O}_{\rightarrow, 0}^{T}$, the complex conjugate of the left eigenvector $\left|v_{n}\right\rangle$ of $\hat{O}_{\rightarrow, 0}$ is the right eigenvector of $\hat{O}_{\leftarrow, 0}$, and vice versa, so that one can assume that

$$
f_{n}(\rho)=v_{n}^{\star}(\rho), \quad g_{n}(\rho)=u_{n}^{\star}(\rho) .
$$

Apart from any degeneracies, the sets of modes can reasonably be assumed to be complete, so that one can formally write

$$
\begin{gathered}
\hat{I}=\sum\left|u_{n}\right\rangle\left\langle v_{n}\left|=\sum\right| f_{n}\right\rangle\left\langle g_{n}\right|, \\
\hat{O}_{\rightarrow, 0}=\sum\left|u_{n}\right\rangle \gamma_{n}\left\langle v_{n}\right|, \\
\hat{O}_{\leftarrow, 0}=\sum\left|f_{n}\right\rangle \gamma_{n}\left\langle g_{n}\left|=\sum\right| v_{n}^{\star}\right\rangle \gamma_{n}\left\langle u_{n}^{\star}\right| .
\end{gathered}
$$

These relations are the formal expressions of Siegman's statement [2] that the adjoint eigenmodes, which we call $\left|f_{n}\right\rangle$, are eigenmodes of the propagation operator in the reversed direction. The biorthogonality relations (3.5) can also be expressed as

$$
\int d \rho f_{n}(\rho) u_{n^{\prime}}(\rho)=\left\langle f_{n}^{\star} \mid u_{n^{\prime}}\right\rangle=\delta_{n n^{\prime}} .
$$

The eigenmodes introduced by Siegman [2] are defined as the eigenvectors of the propagation operator for the lens guide without the amplifying medium, so that the nonunitarity is due to losses only, and the eigenvalues $\gamma_{n}$ have norm smaller than 1. As a slight generalization, we include linear gain in the definition of the propagation operators. When the gain depends on the transverse coordinate $\rho$, it will modify the transverse field distribution in an essential way. During loss action the gain will adapt itself so as to compensate for the losses. This gain clamping will leave the transverse field distribution unchanged only when this additional nonlinear gain is transversely uniform.
A $z$ dependence can be included in the definition of the eigenmodes in a natural way, by allowing propagation from the reference plane $z_{0}$. This gives

$$
\left|u_{n}(z)\right\rangle=\hat{O}_{\rightarrow}\left(z, z_{0}\right)\left|u_{n}\right\rangle,\left|f_{n}(z)\right\rangle=\hat{O}_{\leftarrow}\left(z, z_{0}\right)\left|f_{n}\right\rangle .
$$

Strictly speaking, this defines $\left|u_{n}(z)\right\rangle$ for $z \geqslant z_{0}$ and $\left|f_{n}(z)\right\rangle$ for $z \leqslant z_{0}$. However, provided that the eigenvalue $\gamma_{n}$ is nonzero, the extension to overlapping domains of $z$ is trivial, e.g., by setting $\left|f_{n}(z+2 L)\right\rangle=\left|f_{n}(z)\right\rangle / \gamma_{n}$. Then each mode $\left|u_{n}(z)\right\rangle$ and $\left|f_{n}(z)\right\rangle$ corresponds to a self-reproducing field pattern propagating down the lens guide in the rightward or leftward direction. From Eq. (2.18) it follows that the biorthogonality is conserved during propagation, so that $\int d \rho f_{n}(z, \rho) u_{n^{\prime}}(z, \rho)=\delta_{n n^{\prime}}$ for all values of $z$. When the lens guide represents a standing-wave cavity, the equivalence of the two propagation directions allows us to choose the modes such that

$$
\left|u_{n}(z)\right\rangle=\left|f_{n}(2 L-z)\right\rangle \text {. }
$$

For later convenience we introduce a measure of nonorthogonality of the transverse modes, in the form

$$
Q_{n}(z)=\frac{\left\langle u_{n}(z) \mid u_{n}(z)\right\rangle\left\langle f_{n}(z) \mid f_{n}(z)\right\rangle}{\mid\left\langle f_{n}^{\star}(z)\left|u_{n}(z)\right|^{2}\right.} .
$$

In fact, with the normalization we have chosen in Eq. (3.5) the denominator is unity for all $z$, but for clarity we use here a notation that is independent of normalization. In view of Schwarz's inequality, this factor $Q_{n}$ cannot be smaller than 1 . In the case considered by Siegman [2], $Q_{n}$ coincides with the transverse excess noise factor. As we shall discuss in this paper, this is no longer true for laser media that are nonuniform in the transverse direction, or in the presence of apertures in the cavity. Moreover, in that case, the factor $Q_{n}$ can vary with the longitudinal position $z$. From the propagation equation we find that

$$
\begin{gathered}
\frac{d}{d z}\left\langle u_{n}(z) \mid u_{n}(z)\right\rangle=-\frac{\omega^{2}}{c^{2} k} \int d \rho\left|u_{n}(z, \rho)\right|^{2} \operatorname{Im} \varepsilon, \\
\frac{d}{d z}\left\langle f_{n}(z) \mid f_{n}(z)\right\rangle=\frac{\omega^{2}}{c^{2} k} \int d \rho\left|f_{n}(z, \rho)\right|^{2} \operatorname{Im} \varepsilon .
\end{gathered}
$$

This demonstrates that the quantity $Q_{n}$ as defined in Eq. (3.12) does not vary with $z$ as long as $\operatorname{Im} \varepsilon$ is independent of $\rho$. On the other hand, when $\operatorname{Im} \varepsilon$ is transversely inhomogeneous, $Q_{n}$ generally depends on $z$. By a similar argument, one notices that the factor of $Q_{n}$ will be different on opposite sides of an aperture, when the fractional power loss of the mode $u_{n}$ across the aperture is different from the fractional loss of the counterpropagating mode $f_{n}=v_{n}^{\star}$.

The significance of the factor $Q_{n}$ for noise enhancement can be understood in a simple way. A noise signal is represented by a stochastic field $|p\rangle$, traveling to the right. Expanding the stochastic signal in the eigenmode $\left|u_{n}\right\rangle$ gives the expression 


$$
|p\rangle=\sum\left|u_{n}\right\rangle\left\langle v_{n} \mid p\right\rangle
$$

Assuming that the noise is transversely uniform, the ensemble average of the projection on $|p\rangle$ is proportional to the unit operator so that

$$
\overline{|p\rangle\langle p|}=B \hat{I},
$$

with $B$ a constant measuring the strength of the noise source. This is equivalent to the identity $\overline{p(\rho) p^{\star}\left(\rho^{\prime}\right)}=B \delta\left(\rho-\rho^{\prime}\right)$. When a laser is operating in a single mode $\left|u_{n}\right\rangle$, the other modes are suppressed, and only the contribution $\left|u_{n}\right\rangle\left\langle v_{n} \mid p\right\rangle$ survives in the expansion (3.14). This shows that the noise in the mode $\left|u_{n}\right\rangle$ alone has the strength

$$
\overline{\left\langle p \mid v_{n}\right\rangle\left\langle u_{n} \mid u_{n}\right\rangle\left\langle v_{n} \mid p\right\rangle}=B Q_{n} .
$$

This noise strength is relevant in the case of a laser operating in the mode $\left|u_{n}\right\rangle$ alone. The noise contribution in a single normalized mode $|\phi\rangle$ out of an orthonormal basis would simply be

$$
\overline{\langle\phi \mid p\rangle\langle p \mid \phi\rangle}=B .
$$

This shows that the factor $Q_{n}$ gives the noise enhancement due to the mode nonorthogonality.

The special case of a system that is homogeneous in the propagation direction is described by a Hamiltonian $\hat{H}$ that is independent of $z$. This situation describes pure index or gain guiding. Then the eigenmodes of $\hat{O}_{\rightarrow, 0}$ and $\hat{O}_{\leftarrow, 0}$ are just the eigenmodes of $\hat{H}$, which implies that $\left|u_{n}\right\rangle=\left|f_{n}\right\rangle=\left|v_{n}^{\star}\right\rangle$ $=\left|g_{n}^{\star}\right\rangle$. This is the case originally considered by Petermann [3]. The nonorthogonality factor $Q_{n}$ then coincides with Petermann's excess noise factor (1.2).

\section{SPONTANEOUS-EMISSION NOISE}

Noise induced by spontaneous emission can be modeled as a stochastic dipole polarization $\vec{P}(\vec{r}, t)$, which must be added to the dielectric displacement $\vec{D}$ in Eq. (2.1). The contribution of $\vec{P}$ propagating to the right with the right polarization $\vec{e}$ can be expressed by substituting

$$
\vec{P}(\vec{r}, t) \rightarrow \operatorname{Re} \vec{e} e^{i(k z-\omega t)} P_{\rightarrow}(\vec{r}, t),
$$

with $P_{\rightarrow}(\vec{r}, t)$ slowly varying in space and time. Substitution in Maxwell's equation (2.1) leads to a modified version of Eq. (2.5) in the form

$$
\partial_{z} E_{\rightarrow}=-i \hat{H} E_{\rightarrow}-\frac{\omega}{c^{2} k} \varepsilon_{\mathrm{gr}}(\omega) \partial_{t} E_{\rightarrow}+\frac{i \omega^{2} \mu_{0}}{2 k} P_{\rightarrow} .
$$

We consider the situation of a laser operating in a single transverse mode $\left|u_{0}(z)\right\rangle$. The gain will have adapted itself to the losses in the system, such that the eigenvalue $\gamma_{0}$ has the absolute value 1 , and the wavelength will be such that $\left|u_{n}(z)\right\rangle \exp (i k z)$ is exactly periodical. This defines a $3 \mathrm{D}$ mode

$$
U(\vec{r})=\left\langle\rho \mid u_{0}(z)\right\rangle e^{i k z} .
$$

The noise contribution to the laser light then arises from the projection of the spontaneous-emission polarization (4.1) onto this mode. The corresponding 3D adjoint mode can be constructed from the leftward-propagating mode

$$
F(\vec{r})=\left\langle\rho \mid f_{0}(\vec{r})\right\rangle e^{-i k z} .
$$

Even though we attach no index to these modes, it will be obvious that they are a single member of a generally complete set of 3D modes, each one composed of a transverse eigenmode and a wave number $k$. The set $U(\vec{r})$ is biorthonormal to the set of modes $V(\vec{r})$ that are defined as the complex conjugate of the leftward-propagating mode $F(\vec{r})$. Specifically, from Eqs. (3.5) and (3.6) it follows that

$$
\int d \vec{r} F(\vec{r}) U(\vec{r})=2 L,
$$

where the integration over $z$ extends over one period $2 L$.

When the laser is operating in the single mode $U(\vec{r})$, we can express the field as

$$
E_{\rightarrow}(\vec{r}, t) e^{i k z}=a(t) U(\vec{r}),
$$

where the mode amplitude $a(t)$ is a stochastic quantity as a result of spontaneous emission. Here we use the fact that contributions from the noise to all modes but the lasing one are suppressed during propagation. The evolution equation for Eq. (4.6) is thus given by the projection of Eq. (4.2) on $U(\vec{r})$. Using the propagation equation $(2.10)$ for the mode $\left|u_{0}(z)\right\rangle$ gives for the time derivative of the mode amplitude

$$
d_{t} a(t)=\frac{i \omega}{2 \varepsilon_{0}} \frac{\int d \vec{r} F(\vec{r}) P_{\rightarrow}(\vec{r}) e^{i k z}}{\int d \vec{r} F(\vec{r}) \varepsilon_{\mathrm{gr}}(\omega, \vec{r}) U(\vec{r})} \equiv p_{\rightarrow}(t) .
$$

The stochastic term $P_{\rightarrow}$ in the equation for $p_{\rightarrow}(t)$ models the fluctuations in the electric field due to spontaneous emission events. Since the spontaneous-emission events that are modeled by $P_{\rightarrow}$ can be assumed to be uncorrelated in time and space, we may write

$$
\begin{gathered}
\left\langle P_{\rightarrow}^{\star}\left(\overrightarrow{r^{\prime}}, t^{\prime}\right) P_{\rightarrow}(\vec{r}, t)\right\rangle=B \delta\left(\overrightarrow{r^{\prime}}-\vec{r}\right) \delta\left(t^{\prime}-t\right), \\
\left\langle P_{\rightarrow}(\vec{r}, t)\right\rangle=0,
\end{gathered}
$$

just as for a Langevin force. The value of the function $B$ is given by [2]

$$
B(\vec{r})=\frac{8 \hbar \varepsilon_{0} c}{\omega} b(\vec{r})
$$

where the position-dependent factor

$$
b(\vec{r})=\frac{N_{2}}{N_{2}-N_{1}} g n
$$


contains the intensity gain coefficient $g$, the refractive index $n$, and the occupation numbers $N_{1}$ and $N_{2}$ of the lower and upper state of the lasing transition. This expression also follows from the fluctuation-dissipation theorem [28]. The Langevin properties of $P_{\rightarrow}$ give the identity

$$
\left\langle p^{\star}(t) p\left(t^{\prime}\right)\right\rangle=A \delta\left(t-t^{\prime}\right),
$$

where

$$
A=\left(\frac{\omega}{2 \varepsilon_{0}}\right)^{2} \frac{\int d \vec{r} F^{\star}(\vec{r}) B(\vec{r}) F(\vec{r})}{\left|\int d \vec{r} F(\vec{r}) \varepsilon_{\mathrm{gr}}(\omega, \vec{r}) U(\vec{r})\right|^{2}} .
$$

From Eq. (4.7) we then find

$$
d_{t}\left\langle a^{\star} a\right\rangle=A .
$$

The spontaneous-emission induced laser linewidth can be expressed as [1]

$$
\Delta \omega=\frac{1}{2 W}\left(d_{t} W\right)_{\mathrm{sp}} .
$$

Here we neglect the effect of the instantaneous change in the field intensity due to spontaneous emission on the phase, which means that we assume Henry's $\alpha$ factor to be equal to 0 [29]. For a field characterized by Eq. (4.6), the total field energy in the mode is given by the expression [30]

$$
W=\frac{\varepsilon_{0}}{2}\left\langle a^{\star} a\right\rangle \int d \vec{r} U^{\star}(\vec{r}) U(\vec{r}) \varepsilon_{\mathrm{gr}}(\omega, \vec{r}),
$$

where the integration extends over one full period of the lens guide. The rate of change of the energy $W$ by spontaneous emission is therefore determined by $d_{t}\left\langle a^{\star} a\right\rangle$, which is given in Eq. (4.13). After substitution of the expression (4.12), this gives

$$
\left(d_{t} W\right)_{\mathrm{sp}}=\hbar \omega c \frac{\int d \vec{r} b|F|^{2} \int d \vec{r} \varepsilon_{\mathrm{gr}}|U|^{2}}{\left|\int d \vec{r} \varepsilon_{\mathrm{gr}} F U\right|^{2}} .
$$

This also determines the linewidth (4.14).

Expression (4.15) for the energy also follows by considering the Poynting vector $\vec{S}=\vec{E} \times \vec{H}$, which obeys the identity

$$
-\nabla \cdot \vec{S}=\vec{E} \cdot \partial_{t} \vec{D}+\vec{H} \cdot \partial_{t} \vec{B} .
$$

Expressions for the time derivatives can be obtained from Eq. (2.4) for $n=1$ and a corresponding expression for $\partial_{t} \vec{B}$ [30]. We consider a nonmagnetic material, so that the magnetic permeability is $\mu_{0}$. Furthermore, we assume that

$$
\int d \vec{r} \varepsilon_{0} \varepsilon(\omega) \vec{E}^{2}=\int d \vec{r} \mu_{0} \vec{H}^{2}
$$

for the mode field. This identity is easily justified for modes with negligible losses. After volume integration and averag- ing over the fluctuations, the right-hand side of Eq. (4.17) contains the time derivative of Eq. (4.15). We find

$$
d_{t} W=\mathcal{P}_{\text {gain }}-\int d \vec{r}\langle\nabla \cdot \vec{S}\rangle
$$

where

$$
\mathcal{P}_{\text {gain }}=-\frac{\varepsilon_{0} \omega}{2}\left\langle a^{\star} a\right\rangle \int d \vec{r} U^{\star} U \operatorname{Im} \varepsilon(\omega)
$$

is the net internal power gain. A net loss would make this term negative. Equation (4.19) gives the energy balance of the field in the laser cavity.

Obviously, $\left(d_{t} W\right)_{\mathrm{sp}}$ as expressed by Eq. (4.16) can also be written as $\hbar \omega R$, with $R$ the spontaneous-emission rate into the lasing mode. A similar result for $R$ has been obtained in a different fashion by Champagne and McCarthy [Eq. (20) of Ref. [23]] for the special case of a semiconductor laser with inhomogeneous material coefficients. Our derivation allows for the presence of optical elements such as lenses and apertures anywhere in the cavity. Moreover, we indicated explicitly how the mode and its adjoint should be determined in that case, and what approximations have been made.

\section{UNIFORM MATERIAL PROPERTIES}

\section{A. Fully homogeneous material}

First, we specialize the general result (4.16) for the spontaneous-emission power to the case in which the properties of the medium are homogeneous in the field region. Localized losses can occur at the end mirrors and at apertures that may be positioned anywhere inside the cavity. Then the constant values of $b$ and $\varepsilon_{\mathrm{gr}}$ can be taken out of the integrals in Eq. (4.16). After substituting Eq. (4.10) and using the fact that $c n / \varepsilon_{\text {gr }}$ equals the group velocity $v_{\text {gr }}$, we find

$$
\left(d_{t} W\right)_{\mathrm{sp}}=\hbar \omega g v_{\mathrm{gr}} \frac{N_{2}}{N_{2}-N_{1}} K,
$$

where the excess noise factor $K$ is expressed in terms of the $z$-dependent transverse modes as

$$
K=\frac{\int_{0}^{2 L} d z\langle f(z) \mid f(z)\rangle \int_{0}^{2 L} d z\langle u(z) \mid u(z)\rangle}{\left|\int_{0}^{2 L} d z\left\langle f^{\star}(z) \mid u(z)\right\rangle\right|^{2}} .
$$

The term $g v_{\mathrm{gr}}$ in expression (5.1) represents the relative gain per unit time, which has to be equal to the relative power loss in the steady state. This gives

$$
g v_{\mathrm{gr}}=\mathcal{P}_{0} / W,
$$

with $\mathcal{P}_{0}$ the total power loss. Since $\mathcal{P}_{0} / W$ is commonly defined as the cavity decay rate $\Gamma_{0}$, the intuitive result $\left(d_{t} W\right)_{\mathrm{sp}}=\hbar \omega \Gamma_{0}$ is recovered in the special case where $K$ $=1$ and the inversion is complete. In this case Eq. (4.14) for the linewidth reproduces the Schawlow-Townes expression (1.1). We now proceed to evaluate $K$ in a few specific cases. 


\section{B. Apertures at mirrors}

Next, we consider the situation in which the apertures are located exclusively at the output mirrors. The effective intensity reflectivities of the apertured mirrors are denoted as $R_{1}$ (at $z=L$ ) and $R_{2}$ (at $z=2 L$, which is equivalent to $z=0$ ). When the apertures block part of the lasing mode, these reflectivities are smaller than those of the mirror surfaces, and $R_{1}$ and $R_{2}$ depend on the mode. The gain compensates for the losses at the mirrors and for possible homogeneous internal losses, expressed by the loss coefficient $\kappa$, so that

$$
e^{2 L \alpha} R_{1} R_{2}=1
$$

with $\alpha=g-\kappa$ the difference in gain and loss. Hence, the cavity loss rate is

$$
\Gamma_{0}=g v_{\mathrm{gr}}=\kappa v_{\mathrm{gr}}-\frac{v_{\mathrm{gr}}}{2 L} \ln R_{1} R_{2}
$$

In order to evaluate the excess noise factor (5.2), we express the $z$-depending integrands in terms of their values at the reference plane $z=0^{+}$, just to the right of mirror 2 . We substitute

$$
\begin{gathered}
\langle u(z) \mid u(z)\rangle=e^{\alpha z}\left\langle u\left(0^{+}\right) \mid u\left(0^{+}\right)\right\rangle \\
\langle f(z) \mid f(z)\rangle=e^{-\alpha z}\left\langle f\left(0^{+}\right) \mid f\left(0^{+}\right)\right\rangle \text {for } 0<z<L
\end{gathered}
$$

and

$$
\begin{gathered}
\langle u(z) \mid u(z)\rangle=e^{\alpha z} R_{1}\left\langle u\left(0^{+}\right) \mid u\left(0^{+}\right)\right\rangle \\
\langle f(z) \mid f(z)\rangle=e^{-\alpha z} \frac{1}{R_{1}}\left\langle f\left(0^{+}\right) \mid f\left(0^{+}\right)\right\rangle \quad \text { for } L<z<2 L .
\end{gathered}
$$

Moreover, $\left\langle f^{\star} \mid u\right\rangle$ is independent of $z$, as argued in Sec. III. After these substitutions, the integration over $z$ can be directly performed, with the result

$$
K=K_{T} K_{L}
$$

Here $K_{L}$ is given by Eq. (1.3) and $K_{T}$ is equal to the nonorthogonality measure $Q$ as defined in Eq. (3.12), at the position $z=0^{+}$. In fact, in the present case $Q$ is independent of $z$. Equation (5.8) demonstrates that the excess noise factor factorizes into a transverse and a longitudinal part. The transverse part is sensitive to the phase and amplitude pattern of the mode and the adjoint mode. The longitudinal factor $K_{L}$ is not affected by the phase, and it is determined exclusively by the intensities of the mode and the adjoint mode as a function of the longitudinal coordinate $z$.

\section{Apertures in cavity}

When an aperture is placed somewhere in the cavity, the field in the lens-guide picture passes the aperture twice during one period, once at $z_{0}=0$ and once at $z=2 L-z_{0}$. The situation is sketched in Fig. 2. The loss over one aperture depends on the transverse intensity profile of the mode. For the right-traveling eigenmode $|u\rangle$ we call the effective intensity transmission factors $A_{1}$ at $z_{0}$ and $A_{2}$ at $2 L-z_{0}$. For the adjoint mode $|f\rangle$ (which travels to the left) these factors are $A_{2}$ at $z_{0}$ and $A_{1}$ at $2 L-z_{0}$. As a result, the period $2 L$ is

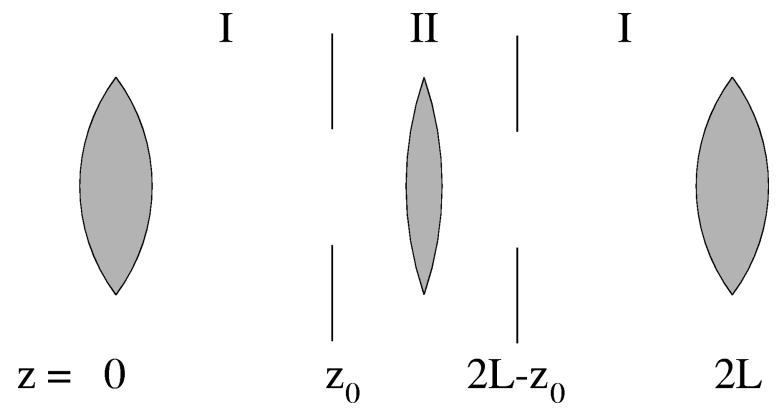

FIG. 2. Equivalent lens guide corresponding to standing-wave cavity with one aperture.

divided in two regions with different values of the nonorthogonality factor $Q$. These are related by

$$
Q_{\mathrm{II}}=\frac{A_{1}}{A_{2}} Q_{\mathrm{I}} \text {, }
$$

where region I contains $z=0$ and region II contains $z=L$. The behavior of the longitudinal field intensities $\langle u(z) \mid u(z)\rangle$ and $\langle f(z) \mid f(z)\rangle$ as a function of $z$ is fully determined by the intensity factors $A_{1}$ and $A_{2}$ across the apertures and $R_{1}$ and $R_{2}$ at the mirrors, combined with the exponential behavior $\sim \exp ( \pm \alpha z)$ in between. Using Eq. (5.9), the excess noise can be expressed as a product of the factor $Q$ in one of the regions times an integral expression over the $z$ dependence of the longitudinal field intensities. In this way, $K$ can be factorized in a transverse contribution $Q$ and an effective longitudinal term.

As an example, we consider the case that appreciable losses occur only at the apertures, so that $R_{1}=R_{2}=1$ and $\exp (-2 \alpha L)=A_{1} A_{2}$. In this case, Eq. (5.2) for the factor $K$ can be written as

$$
K=Q_{\mathrm{I}}^{z_{0} / L} Q_{\mathrm{II}}^{1-z_{0} / L} K_{L},
$$

with

$$
\begin{aligned}
K_{L}= & \frac{1}{\ln ^{2}\left(A_{1} A_{2}\right)}\left[A_{1}^{z_{0} / L}\left(\frac{1}{\sqrt{A_{1} A_{2}}}-\sqrt{\frac{A_{2}}{A_{1}}}\right)\right. \\
& \left.+A_{2}^{1-z_{0} / L}\left(\frac{1}{\sqrt{A_{1} A_{2}}}-\sqrt{\frac{A_{1}}{A_{2}}}\right)\right]^{2} .
\end{aligned}
$$

In Eq. (5.10), the term $K_{L}$ can be viewed as an effective longitudinal excess noise factor, in which $A_{1}$ and $A_{2}$ appear in a symmetric way. The two factors $Q_{\mathrm{I}}$ and $Q_{\mathrm{II}}$ contribute to the effective transverse noise factor $Q_{\mathrm{I}}^{z_{0} / L} Q_{\mathrm{II}}^{1-z_{0} / L}$, in accordance with the size of the two regions.

It is natural to compare $K_{L}$ as given in Eq. (5.11) to the corresponding longitudinal factor $K_{L \text {,hom }}$ for a lens guide with two homogeneous absorbers located at $z_{0}$ and $2 L$ $-z_{0}$, with intensity transmittivities $A_{1}$ and $A_{2}$. In both locations, the transmittivity is the same for the mode and its adjoint. In Fig. 3 the ratio $K_{L} / K_{L \text {,hom }}$ is plotted as a function of $z_{0} / L$ and $\ln \left(A_{1} / A_{2}\right)$. For realistic values, this ratio deviates only slightly from 1 . Notice that the longitudinal field intensity $\langle u(z) \mid u(z)\rangle$ of the mode is the same in these two cases, whereas the factors $\langle f(z) \mid f(z)\rangle$ for the adjoint modes are 


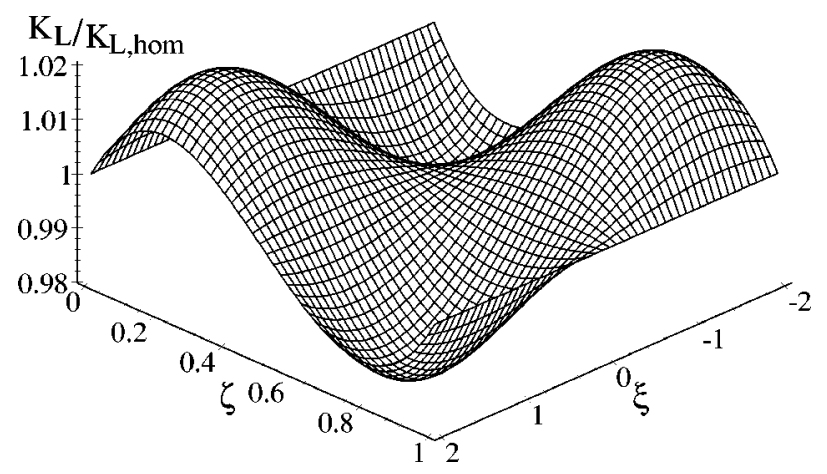

FIG. 3. Ratio $K_{L} / K_{L \text {,hom }}$ between longitudinal noise factors for cavity with aperture and equivalent lens guide with homogeneous absorbers, as a function of position and transmittivity ratio; $\zeta$ $=z_{0} / L, \quad \xi=\ln \left(A_{1} / A_{2}\right)$. The total loss factor is taken as $A_{1} A_{2}$ $=0.09$.

different. This shows that for two different situations with the same longitudinal mode structure, the adjoint modes are not necessarily the same.

The longitudinal noise factor (5.11) reduces to the standard expression (1.3) in two special cases. When $z_{0}=L$, the aperture is located at a mirror. Then $K$ can be written as $K$ $=Q_{I} K_{L}$, where $K_{L}$ coincides with Eq. (1.3), with $R_{1}=1$ and $R_{2}=A_{1} A_{2}$. A second special case occurs when $z_{0}=L / 2$. Then Eq. (5.11) is identical to Eq. (1.3) for $K_{L}$ with $R_{i}$ replaced by $A_{i}$. For $z_{0}=L / 2$, the transverse excess noise factor $K_{T}$ equals $\sqrt{Q_{\mathrm{I}} Q_{\mathrm{II}}}$.

This situation demonstrates that in a standing-wave cavity with a single aperture, the two regions between the mirrors separated by the aperture generally have different values of the nonorthogonality measure $Q$, and thereby of the transverse excess noise factor. On the other hand, in a ring laser, where the period $2 L$ spans one cycle of the ring, at least two apertures are needed to create two separate regions with different $Q$ values.

\section{Transversely homogeneous material properties}

When the properties of the laser medium are inhomogeneous, it is no longer possible to extract an unambigous excess noise factor $K$ from Eq. (4.16) as we did in Eq. (5.1). However, when the material properties vary only with the longitudinal coordinate $z$, a factorization of the noise term in a transverse and a longitudinal contribution is still possible. Equation (4.16) then gives

$$
\left(d_{t} W\right)_{\mathrm{sp}}=\hbar \omega c \frac{\int d z b(z)\langle f(z) \mid f(z)\rangle \int d z \varepsilon_{\mathrm{gr}}(z)\langle u(z) \mid u(z)\rangle}{\left|\int d z \varepsilon_{\mathrm{gr}}(z)\left\langle f^{\star}(z) \mid u(z)\right\rangle\right|^{2}} .
$$

The $z$-dependent intensity of the mode can be expressed in terms of a periodic function $G$, defined by

$$
\langle u(z) \mid u(z)\rangle=G(z)\left\langle u\left(0^{+}\right) \mid u\left(0^{+}\right)\right\rangle .
$$

When the apertures are located only at the mirrors, the corresponding relation for the adjoint mode reads

$$
\langle f(z) \mid f(z)\rangle=\frac{1}{G(z)}\left\langle f\left(0^{+}\right) \mid f\left(0^{+}\right)\right\rangle
$$

and the quantity $Q$ is independent of the longitudinal coordinate $z$. Then Eq. (5.12) gives

$$
\left(d_{t} W\right)_{\mathrm{sp}}=\hbar \omega c Q \frac{\int d z b(z) / G(z) \int d z \varepsilon_{\mathrm{gr}}(z) G(z)}{\left|\int d z \varepsilon_{g r}(z)\right|^{2}} .
$$

In this expression the nonorthogonality factor $Q$ still plays the role of a transverse excess noise factor.

A single aperture in the cavity at the position $z_{0}$ divides the period into two regions with possibly different values of $Q_{\mathrm{I}}$ and $Q_{\mathrm{II}}$, just as in the case of a fully homogeneous medium. Moreover, when the function $G(z)$ is still defined by Eq. (5.13), one easily checks that

$$
\begin{aligned}
\langle f(z) \mid f(z)\rangle & =\frac{1}{G(z)}\left\langle f\left(0^{+}\right) \mid f\left(0^{+}\right)\right\rangle \quad \text { for } \quad z \in \mathrm{I}, \\
& =\frac{A_{1}}{A_{2}} \frac{1}{G(z)}\left\langle f\left(0^{+}\right) \mid f\left(0^{+}\right)\right\rangle \quad \text { for } \quad z \in \mathrm{II} .
\end{aligned}
$$

When the gain medium is located exclusively in region I, the first integral in the numerator of Eq. (5.12) only extends over this part of the period $2 L$. In this case, the result (5.15) still holds, with $Q$ replaced by $Q_{\mathrm{I}}$. The transverse excess noise is then determined by the value of the quantity $Q$ in the gain region.

\section{CONCLUSION}

We derived a general expression for the linewidth of a single-mode laser induced by spontaneous emission. We allow for localized losses both at mirrors and at apertures, and for nonuniform properties of the laser medium. Spontaneous emission is modeled as a classical fluctuating dipole polarization. The key result is given by Eq. (4.16), which represents the spontaneous-emission power into the lasing mode. This determines the linewidth (4.14). The three-dimensional adjoint mode $F$ is explicitly specified for any given laser mode $U$. 
A result with a structure similar to Eq. (4.16) has been obtained in Ref. [23] for semiconductor lasers with axially varying material properties. Our treatment allows for apertures either at the mirrors or inside the cavity, which can give rise to unstable cavities, and strong noise enhancement. It has been shown before that for homogeneous medium properties the nonorthogonality of the transverse modes gives rise to enhancement of laser noise [3,2]. This enhancement is given by the quantity (3.12), which is a measure of the nonorthogonality of the transverse modes. For transversely inhomogeneous material properties, and in the presence of apertures, this quantity $Q$ can depend on the longitudinal coordinate $z$, which makes the combination of transverse and longitudinal noise enhancement a delicate problem.

When the material properties are uniform in the transverse direction, the laser noise factorizes into a transverse enhancement factor, given by $Q$, and a longitudinal factor, which involves integrals over the $z$-dependent beam intensity only. This factorization, expressed in Eq. (5.15), remains true when in a standing-wave laser, apertures are positioned at one or both mirrors. However, when an aperture divides the cavity into two regions, the value of the transverse enhancement factor $Q$ is usually different in these regions. The total noise enhancement can then be written as the product of a longitudinal factor and an effective transverse factor. When the laser medium is uniform, this separation is given in Eq. (5.10). When the aperture is located in the middle of the cavity, the transverse excess noise factor is just the geometric mean of the factors $Q$ pertaining to the two regions. When the gain occurs in one region only, the same factorized expression (5.15) remains valid, with the transverse factor $Q$ taken in the gain region.

\section{ACKNOWLEDGMENTS}

This work is part of the research program of the Stichting voor Fundamenteel Onderzoek der Materie (FOM), which is supported by the NWO.
[1] A. L. Schawlow and C. H. Townes, Phys. Rev. 112, 1940 (1958).

[2] A. E. Siegman, Phys. Rev. A 39, 1253 (1989).

[3] K. Petermann, IEEE J. Quantum Electron. QE-15, 566 (1979).

[4] G. H. C. New, J. Mod. Opt. 42, 799 (1995).

[5] M. A. Rippin and G. H. C. New, J. Mod. Opt. 43, 993 (1996).

[6] M. A. van Eijkelenborg, A. M. Lindberg, M. S. Thijssen, and J. P. Woerdman, Phys. Rev. Lett. 77, 4314 (1996).

[7] M. Brunel, G. Ropars, A. Le Floch, and F. Bretenaker, Phys. Rev. A 55, 4563 (1997).

[8] O. Emille, M. Brunel, F. Bretenaker, and A. Le Floch, Phys. Rev. A 57, 4889 (1998).

[9] Å. M. Lindberg, M. A. van Eijkelenborg, K. Joosten, G. Nienhuis, and J. P. Woerdman, Phys. Rev. A 57, 3036 (1998).

[10] C. H. Henry, J. Lightwave Technol. LT-4, 288 (1986).

[11] P. Goldberg, P. W. Milonni, and B. Sundaram, Phys. Rev. A 44, 1969 (1991).

[12] I. H. Deutsch, J. C. Garrison, and E. M. Wright, J. Opt. Soc. Am. B 8, 1244 (1991).

[13] W. A. Hamel and J. P. Woerdman, Phys. Rev. A 40, 2785 (1989).

[14] K. Ujihara, IEEE J. Quantum Electron. QE-20, 814 (1984).

[15] M. P. van Exter, S. J. M. Kuppens, and J. P. Woerdman, Phys. Rev. A 51, 809 (1995).
[16] A. Tyszka-Zawadzka, P. Szczepański, and A. Kujawski, Opt. Lett. 20, 881 (1995).

[17] W. A. Hamel and J. P. Woerdman, Phys. Rev. Lett. 64, 1506 (1990).

[18] Y. J. Cheng, P. L. Mussche, and A. E. Siegman, IEEE J. Quantum Electron. 30, 1498 (1994).

[19] Y. J. Cheng, C. G. Fanning, and A. E. Siegman, Phys. Rev. Lett. 77, 627 (1996).

[20] M. A. van Eijkelenborg, M. P. van Exter, and J. P. Woerdman, Phys. Rev. A 57, 571 (1998).

[21] J. Arnaud, Opt. Quantum Electron. 18, 335 (1986).

[22] K. Petermann, Laser Diode Modulation and Noise (Kluwer Academic Publishers, Dordrecht, 1988).

[23] Y. Champagne and N. McCarthy, IEEE J. Quantum Electron. 28, 128 (1992).

[24] A. G. Fox and T. Li, Bell Syst. Tech. J. 40, 453 (1961).

[25] A. E. Siegman, Phys. Rev. A 39, 1264 (1989).

[26] P. W. Milonni, J. Mod. Opt. 42, 1991 (1995).

[27] D. Stoler, J. Opt. Soc. Am. B 71, 334 (1981).

[28] C. H. Henry and R. F. Kazarinov, Rev. Mod. Phys. 68, 801 (1996).

[29] C. H. Henry, IEEE J. Quantum Electron. QE-18, 259 (1982).

[30] L. D. Landau and E. M. Lifshitz, Electrodynamics of Continuous Media (Pergamon Press, Oxford, 1960), Chap. 63. 